\section{Are NICE guidelines losing their impartiality?}

I applaud Taylor \& Perera ${ }^{1}$ for their clear discussion of these very important issues. For me the most important sentence in their piece is the last one, that 'CG178 appears to be open to a critique of bias'. This is not the first occasion that such issues have arisen and I think that it is time for the National Institute for Health and Care Excellence (NICE) to take a long hard look at the relative standards that are set for making recommendations about the use of non-pharmacological and pharmacological treatments. A previous example is seen in CG72 Attention Deficit Hyperactivity Disorder, ${ }^{2}$ where it would appear that a lower quality of trials was allowed and lower standards of evidence were required to support behavioural approaches than for pharmacological treatments. A similar criticism can be made about CG28 Depression in Children and Young People, ${ }^{3}$ and there are no doubt others. Although the ultimate recommendations made in these guidelines may, on one level at least, be sensible, I believe that the evaluation and interpretation of the evidence, including the selection of trials and assessment of their quality as well as their outcomes, should be the same regardless of the mode of treatment. If NICE, who as Taylor \& Perera point out occupy an extremely important position in our lives, then decide to interpret or weight evidence differently, this should be clear and transparent. NICE must be above all claims of bias and need to work hard to ensure that they regain this position.

1 Taylor M, Perera U. NICE CG178 Psychosis and Schizophrenia in Adults: Treatment and Management - an evidence-based guideline? Br J Psychiatry 2015; 206: 357-9.

2 National Institute for Health and Clinical Excellence. Attention Deficit Hyperactivity Disorder: Diagnosis and Management of ADHD in Children, Young People and Adults (Clinical Guideline CG72). NICE, 2008.

3 National Institute for Health and Clinical Excellence. Depression in Children and Young People: Identification and Management in Primary, Community and Secondary Care (Clinical Guideline CG28). NICE, 2005

\section{Declaration of interest}

D.C. has accepted fees and/or research funding from Janssen Cilag, Shire, Lilly, Lundbeck, Novartis, and Vifor in the past 3 years. He receives royalties from Oxford University Press.

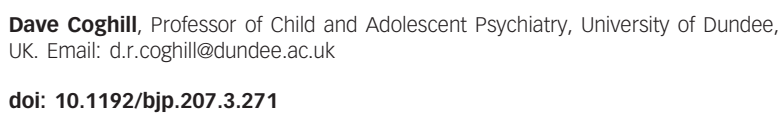

doi: 10.1192/bjp.207.3.271

Authors' reply: We thank Professor Coghill for his interest in our editorial, and his positive comments supporting our views. It is notable that, thus far at least, the only comments either of us authors have received have been positive despite our editorial being deliberately tendentious, and what can appear to be a worldwide promotion of cognitive-behavioural therapy for psychosis. A related point is that even if cognitive-behavioural therapy for psychosis was in fact highly efficacious, there seems to be such a dearth of clinical psychologists in the National Health Service that accessing even an initial assessment can take up to 18 weeks - a long time to wait for someone actively psychotic.

\section{Declaration of interest}

M.T. was chair of SIGN 131 guideline, and has received fees and/or hospitality from Janssen, Lundbeck, Roche, and Sunovion in the past 3 years.

Mark Taylor, Royal Edinburgh Hospital, Edinburgh, UK. Email: marktaylor2@nhs.net; Udayanga Perera, District General Hospital, Monaragala, Sri Lanka

doi: $10.1192 /$ bjp.207.3.271a

\section{Hearing voices: are we getting the message?}

In a large study of adults with bipolar disorder, Upthegrove and colleagues report associations between childhood sexual abuse and lifetime occurrence of mood congruent auditory and visual hallucinations; however, no associations are seen for delusions or diagnoses of psychotic disorders. ${ }^{1}$ The findings are similar to a recent study of psychotic symptoms in borderline personality disorder (BPD) that shows high lifetime prevalence of auditory and other hallucinations (with predominantly negative contents) but not delusions. ${ }^{2}$ Together these studies provide important clues regarding mechanisms of specific psychopathology. They also raise a wider question regarding the relationships between psychotic and common mental symptoms such as mood and anxiety.

Using interviews with the Present State Examination, the BPD study $^{2}$ found that $80 \%$ of 30 patients (collected from a specialist personality disorder service) had experienced psychotic symptoms at some point during their lifetime. Auditory hallucinations were reported by $50 \%$ and visual hallucinations were present in about a third of the sample. Although the form of auditory hallucinations was similar to that in schizophrenia, the content was predominantly negative and critical even when they occurred outside an affective episode. Contents of visual and olfactory hallucinations were also mainly negative and unpleasant. Delusions, however, when present, indicated previously undiagnosed psychotic disorder. Although the study did not examine maltreatment specifically, such history is common in BPD. Thus mood dysregulation, which is an important feature of both BPD and bipolar disorder, might explain the emergence of negative, self-critical auditory/visual/other hallucinations in victims of childhood maltreatment.

The findings along with other research indicate psychotic symptoms are common and can occur in the context of nonpsychotic disorders. A recent phenomenological study found that auditory hallucinations are present in a diverse sample of people with various diagnoses and clinical histories, where they are associated with fear, anxiety, depression and stress as well as positive or neutral emotions. ${ }^{3}$ In young people, auditory hallucinations have been reported to occur alongside mild to moderate depression and anxiety, where they are a marker of severity, for example multiple psychiatric comorbidity or suicidality. ${ }^{4}$ Similarly, a recent study found that depression, anxiety and psychotic symptoms measure a single, common underlying factor in the population, with psychotic items measuring the more severe end of this continuum. ${ }^{5}$ Together these findings suggest that similar to depression and anxiety, psychotic symptoms - particularly auditory hallucinations - are common mental symptoms. Therefore, psychotic phenomena should be routinely included in epidemiological assessments of psychiatric morbidity. Diagnostic classification systems should acknowledge the presence of psychotic symptoms in non-psychotic disorders to reflect evidence, which will also allay worries among patients and many clinicians who tend to associate hallucinations exclusively with psychosis.

1 Upthegrove R, Chard C, Jones L, Gordon-Smith K, Forty L, Jones I, et al. Adverse childhood events and psychosis in bipolar affective disorder. Br J Psychiatry 2015; 206: 191-7.

2 Pearse L, Dibben C, Ziauddeen H, Denman C, McKenna PJ. A study of psychotic symptoms in borderline personality disorder. J Nerv Ment Dis 2014; 202: $368-71$.

3 Woods A, Jones N, Alderson-Day B, Callard F, Fernyhough C. Experiences of hearing voices: analysis of a novel phenomenological survey. Lancet Psychiatry 2015; 4: 232-31.

4 Kelleher I, Keeley H, Corcoran P, Lynch F, Fitzpatrick C, Devlin N, et al. Clinicopathological significance of psychotic experiences in non-psychotic young people: evidence from four population-based studies. Br J Psychiatry 2012; 201: 26-32. 
5 Stochl J, Khandaker GM, Lewis G, Perez J, Goodyer IM, Zammit S, et al. Mood, anxiety and psychotic phenomena measure a common psychopathological factor. Psychol Med 2015; 45: 1483-93.

Golam M. Khandaker, Clinical Lecturer, Department of Psychiatry, University of Cambridge, UK. Email: gmk24@medschl.cam.ac.uk; Claire R. M. Dibben, Consultan Psychiatrist, Norfold and Suffolk NHS Foundation Trust, Bury St Edmunds, UK; Peter B. Jones, Professor, Department of Psychiatry, University of Cambridge, UK

doi: 10.1192/bjp.207.3.271b

Authors' reply: We thank Khandaker et al for their response to our paper, and are in broad agreement that psychotic symptoms are more common than currently recognised in mood disorders. Indeed our previous work has highlighted this and likewise the importance of mood symptoms in psychosis. ${ }^{1,2}$ In our present study the presence of hallucination but not delusion was most frequent in those with a history of child sexual abuse. ${ }^{3}$ Hallucinations in particular may occur in non-psychotic diagnoses in the presence of childhood or later trauma. ${ }^{3}$ Thus hallucinations may have less specificity for psychosis than one might expect given the weight positive symptoms have in diagnostic terms. ${ }^{4,5}$ Khandaker et al's proposal that diagnostic systems should acknowledge the presence of these experiences in non-psychotic disorders is important. This recognition would allow clinicians to better accept diagnostic uncertainty, allay worries provoked by an exclusive association of hallucinations with psychosis, and enable further investigation as to underlying causality within a symptom-specific approach.

1 Hamshere ML, Green EK, Jones IR, Jones L, Moskvina V, Kirov G, et al. Genetic utility of broadly defined bipolar schizoaffective disorder as a diagnostic concept. Br J Psychiatry 2009; 195: 23-9.

2 Upthegrove R, Ross K, Brunet K, McCollum R, Jones L. Depression in firs episode psychosis: the role of subordination and shame. Psychiatry Res 2014; 217: 177-84.

3 Upthegrove $\mathrm{R}$, Chard $\mathrm{C}$, Jones $\mathrm{L}$, Gordon-Smith $\mathrm{K}$, Forty $\mathrm{L}$, Jones I, et al. Adverse childhood events and psychosis in bipolar affective disorder Br J Psychiatry 2015; 206: 191-7.

4 Larøi F, Sommer IE, Blom JD, Fernyhough C, Hugdahl K, Johns LC, et al. The characteristic features of auditory verbal hallucinations in clinical and nonclinical groups: state-of-the-art overview and future directions. Schizoph Bull 2012; 38: 724-33.

5 Fusar-Poli P, Nelson B, Valmaggia L, Yung AR, McGuire PK. Comorbid depressive and anxiety disorders in 509 individuals with an at-risk mental state: impact on psychopathology and transition to psychosis. Schizophr Bull 2014; 40: 120-31.

Rachel Upthegrove, Department of Psychiatry, School of Clinical \& Experimental Medicine, University of Birmingham, Birmingham, Bipolar Disorder Research Network and Early Intervention Service, Birmingham and Solihull Mental Health Foundation Trust, Birmingham, UK Email: R.Upthegrove@bham ac uk: Ian Jones, Nick Craddock, National Centre for Mental Health, MRC Centre for Neuropsychiatric Genetics and Genomics, Cardiff University, Cardiff and Bipolar Disorder Research Network, UK

doi: 10.1192/bjp.207.3.272

\section{Childhood non-affective psychoses: data analysis}

The epidemiology of psychosis in children is a story of even smaller numbers than for psychosis in grown-ups. But how much smaller? The short report of Tiffin \& Kitchen ${ }^{1}$ on incident cases of schizophrenia in children in 2010-2011 is a well-needed addition to a limited descriptive literature on psychosis in children. I have a couple of points about the analysis, which I feel might clarify the report.

The authors point out at the outset the heuristic that earlier onset cases of a disorder might have a 'greater loading of causal factors'. This does not seem to be correct - all cases of a given disorder represent the interrelation of a number of risk factors on a causal pathway - it is therefore unclear why earlier cases would represent the culmination of pathways with a greater number of causal factors. The authors may mean that earlier onset psychoses could represent individuals with stronger risk factors for the disorder. However, although this is usually correct for diseases in general, this point rests on the assumption that there is a single underlying outcome under study, which has long been contested. ${ }^{2}$ Assuming this heterogeneity is related in some way to the clinical heterogeneity of schizophrenia across people of different ages and with different risk factor patterns, this may explain the large number of false positives identified in Tiffin \& Kitchen's study.

Second, although the authors present confidence intervals (presumably based on Poisson standard errors), it is important to point out what the intervals are referring to - this study attempts to capture all cases within a surveillance approach, rather than to sample the target population. In this case, the confidence intervals refer to the underlying randomness of the disease process, rather than to the design of the study. ${ }^{3}$ Confidence intervals used in this way are not meaningless, but they may not be strictly necessary here - it seems that reporting the crude rate in this type of study is an almost equally informative approach for the purposes of this research. Furthermore, uncertainty about the true rate of the disorder due to random variability ('error') in the disease process likely pales in comparison with the systematic error introduced by biases in this design.

1 Tiffin PA, Kitchen CEW. Incidence and 12-month outcome of childhood nonaffective psychoses: British national surveillance study. Br J Psychiatry 2015; 206: $517-8$.

2 Tsuang MT, Lyons MJ, Faraone SV. Heterogeneity of schizophrenia. Conceptual models and analytic strategies. Br J Psychiatry 1990; 156 $17-26$.

3 Eayres D. Technical Briefing 3: Commonly Used Public Health Statistics and their Confidence Intervals. Association of Public Health Observatories, 2008.

Vishal Bhavsar, Epidemiologist, Institute of Psychiatry, Psychology and Neuroscience, King's College London, UK. Email: vishal.2.bhavsar@kcl.ac.uk

doi: 10.1192/bjp.207.3.272a

Authors' reply: To clarify, we did mean to state that earlier onset psychoses could represent individuals with stronger risk factors for the disorder. We agree that there is likely to be some heterogeneity in the outcomes, even if the loading of putative causal factors is similar for any set of given individuals. However, there is evidence that cases of childhood-onset psychosis spectrum disorders in general, when carefully defined, tend to be more severe and more homogeneous, with stronger family histories of schizophrenia spectrum disorders than adult-onset cases. ${ }^{1-3}$ Therefore, there may still be much to be learned about causality, even if the assumption of homogeneous clinical outcomes does not hold strictly true. Moreover, we believe the high rate of falsepositive reports is likely to be due, at least in most instances, to clinicians initially wrongly attributing perceptual disturbance in children to an underlying psychotic illness. In most cases, it is likely that voice experiences and other potentially psychotic phenomena may result from processes that may be conceptualised as more psychologically driven, such as dissociation. Such experiences are commonly reported in community samples of children and adolescents, who are likely to share few, if any, of the risk factors associated with the development of early-onset schizophrenia spectrum disorders. ${ }^{4}$ 